\title{
Segmented Monolithic Germanium Detector Arrays for X-ray Absorption Spectroscopy
}

The work in Phase I experimentally demonstrated an innovative detector fabrication technique suitable for making multi-element monolithic X-ray detectors. As this technique proved successful during Phase I, a Phase II application was submitted to pursue full XAS system development.

Multi-element germanium planar detector technology is our specialty. We strive to bring innovative planar detector processing techniques to bear on germanium detector technology development. The appropriate germanium planar detector fabrication technology was investigated to understand its potential to provide better XAS detector arrays.

The experimental results from the Phase I effort were extremely encouraging. During Phase I PHDs Co. made the first strides toward a new detector technology that could have great impact on synchrotron $\mathrm{x}$-ray absorption (XAS) measurements, and x-ray detector technology in general. Detector hardware that allowed critical demonstration measurements of our technology was designed and fabricated. This new technology allows good charge collection from many pixels on a single side of a multi-element monolithic germanium planar detector. The detector technology provides "dot-like" collection electrodes having very low capacitance. The detector technology appears to perform as anticipated in the Phase I proposal. In particular, the 7pixel detector studied showed remarkable properties; making it an interesting example of detector physics. The technology is enabled by the use of amorphous germanium contact technology on germanium planar detectors. Because of the scalability associated with the fabrication of these technologies at PHDs Co., we anticipate being able to supply larger detector systems at significantly lower cost than systems made in the conventional manner.

There were three major tasks performed during Phase I. First, a liquidnitrogen cooled test-cryostat was modified to allow low energy photons to impinge on the detectors. Secondly, measurements were made on ordinary guard-ring planar detectors. Finally, measurements were made on a 7-pixel xray detector array that had very low capacitance but good charge collection. The 7-pixel detector measurements serve as the most compelling data to support the viability of the technology for XAS detectors.

One of our test-detector cryostats was modified to facilitate the measurement of low energy photons. This modification included installation of beryllium vacuum windows on the top of the test cryostat. In addition, modified detector holders were designed and fabricated to hold the detectors upside down inside the cryostat. These modifications allow low-energy photons to impinge on the bias electrode of the detector through the beryllium windows. Until this project, most of our projects concentrated on higher-energy gamma-ray detection. Consequently, our detectors are usually held and tested in relatively thick aluminum cryostats with little concern for the detector orientation. The four 
beryllium windows were 0.005 " thick. The windows were set in epoxy inside a hole having a countersink.

Inside the test cryostat the detectors are held in an upside-down detector mount that allows photons to impinge on the bias electrode of the detector. The mechanical mounting structure was tested using a set of 4 of our planar test detectors. These germanium planar detectors are made using amorphous germanium detector technology. The metal contacts are evaporated over a sputtered amorphous-germanium layer to form a rectifying contact. On the collection side of the detector, the aluminum contact is segmented into center and guard ring segments. Normally these contacts are face-up in our test cryostat. For this x-ray work, we needed the bias contact to be face up, so this mounting structure was designed and fabricated. The planar test detectors are held in place with three spring-loaded pogo pins. The guard ring segment is used to prevent intrinsic-surface leakage current from causing noise in the center contact.

The first measurements were made with the four simple planar detectors. The detectors were mounted in the test cryostat. These detectors were all 2.3$\mathrm{mm}$ thick and $29-\mathrm{mm}$ diameter. The detectors were fabricated from extremely pure n-type germanium, $\sim 2.2 \times 10^{9} / \mathrm{cm}^{3}$. All four detectors showed good transmission of low energy photons through the beryllium cryostat windows and the bias electrode of the detector. The $5.9-\mathrm{keV} x$ rays from ${ }^{55} \mathrm{Fe}$ were visible in the energy spectrum. The $14.4-\mathrm{keV}$ gamma-ray peak from ${ }^{57} \mathrm{Co}$ had a FWHM = $940 \mathrm{eV}$ at $4-\mu \mathrm{s}$ peaking time. This rather high noise is due to the detector capacitance $\sim 15 \mathrm{pF}$ and the capacitance of the JFET $\sim 10 \mathrm{pF}$. The preamplifier used here was designed for higher energy gamma-ray measurements. Even with these relatively large noise values, we were still able to evaluate the benefits of the monolithic $x$-ray planar detector technology. For this measurement, a detector was biased to $-100 \mathrm{~V}$. These measurements clearly demonstrated the viability of the detector technology using amorphous germanium contacts. Photons interacting at a relatively shallow depth $(1 / \mathrm{e} \sim 17 \mu \mathrm{m}$ for $14.4-\mathrm{keV}$ photons in germanium) on the bias side of the detector create charge carriers that are collected by the collecting electrode.

It was very encouraging to observe functionality in an ordinary planar detector. However, one of our Phase I reviewers correctly noted: "It is much harder to produce a correctly functioning test detector than the final array." The geometry of these planar detectors was certainly not ideal. This is especially true considering that our test detectors were only $2.3-\mathrm{mm}$ thick. The main purpose of these measurements with the planar test detectors was to insure a working system.

With these thoughts in mind, we turned our attention to the 7-pixel detector designed with a much different geometry. The 7-pixel detector was expected to segment into a roughly hexagonal pattern because of the hexagonal pattern of the pixels. The detector was made in the same fashion as the planar detectors described above, except that a precision shadow mask was used to perform the aluminum evaporation over the amorphous germanium. A special 0.005 " thick stainless-steel mask was designed and photo lithographically fabricated to 
produce the desired electrode pattern. The mask was designed to produce the pattern with a series of two complimentary aluminum evaporations. The evaporation established small (2.5-mm diameter) dot electrodes. The small dotlike collection electrodes should have an input capacitance of $\sim 1 \mathrm{pF}$, allowing low noise detector pixels to be operated at relatively short peaking times $\sim 1 \mu \mathrm{s}$.

The detector was fabricated and loaded into the cryostat. Like the planar test detectors, the bias electrode faced the Be windows to allow low-energy photon transmission into the detector. Connection was made to each of the 7 collection electrodes by a set of 7 pogo pins captured in a circular block of Teflon with a small piece of indium foil on each tip. Each collection electrode was connected to a PHDs Co. charge sensitive preamplifier outside the cryostat. The mask for placing and fabricating the collection-electrode side of the detector keys into one of the three support beams in the detector holder. The detector was fabricated from a 32-mm diameter, 4-mm thick piece of $\mathrm{n}$-type germanium having an impurity concentration of $\sim 1.1 \times 10^{10} / \mathrm{cm}^{3}$.

Once at liquid nitrogen temperature, the detector was biased. The detector depleted at a bias voltage of $\sim-90 \mathrm{~V}$. Operating in this mode, a very small count rate of $5.9-\mathrm{keV}$ and $122-\mathrm{keV}$ photons was visible from each of the seven pixels of the detector. A voltage pulser signal was presented to the bias electrode of the detector through the last filtering capacitor $(8 \mathrm{nF})$ of the high voltage filter box. Using this voltage pulser, an equivalent charge signal is measured out of the preamplifier attached to each of the seven pixels. The pulse height of this resulting charge signal was calibrated into energy using gamma-ray signals. From energy, the signal can be converted into charge using the value of $\varepsilon=2.96$ $\mathrm{eV} / 1.6 \times 10^{-19}$ Coulombs. A known voltage pulse resulting in a measured charge corresponds to the capacitance of the collection electrode relative to the bias electrode of the detector. The capacitance of each pixel was measured in this manner and found to be $1.0 \mathrm{pF}$. The pixel capacitance did not change once the detector was fully depleted. In the Phase I proposal we had calculated a collection-electrode capacitance of $1 \mathrm{pF}$ for our 7-pixel detector design. This capacitance is low enough to make an excellent XAS detector. The collectionelectrode capacitance is roughly proportional to the diameter of the dot-like collection electrodes. These dots are $2.5-\mathrm{mm}$ diameter. The dots could be made smaller in the future to match a smaller capacitance JFET if this strategy seems appropriate considering all the other instrumentation boundary conditions (readout electronics, cryostat, etc.). Lower capacitance collection electrodes can be fabricated using a shadow mask having smaller holes to make the collection electrodes. Currently, our preamplifiers have 10-pF JFETs operating at room temperature so the JFET capacitance dominates the noise at short peaking times. On the 7-pixel detector we measured noise of $F W H M=950 \mathrm{eV}$ at a peaking time of $1 \mu \mathrm{s}$ and $\mathrm{FWHM}=750 \mathrm{eV}$ at a peaking time of $4 \mu \mathrm{s}$. This noise level provided sufficiently good spectroscopy for the demonstration measurements here. 


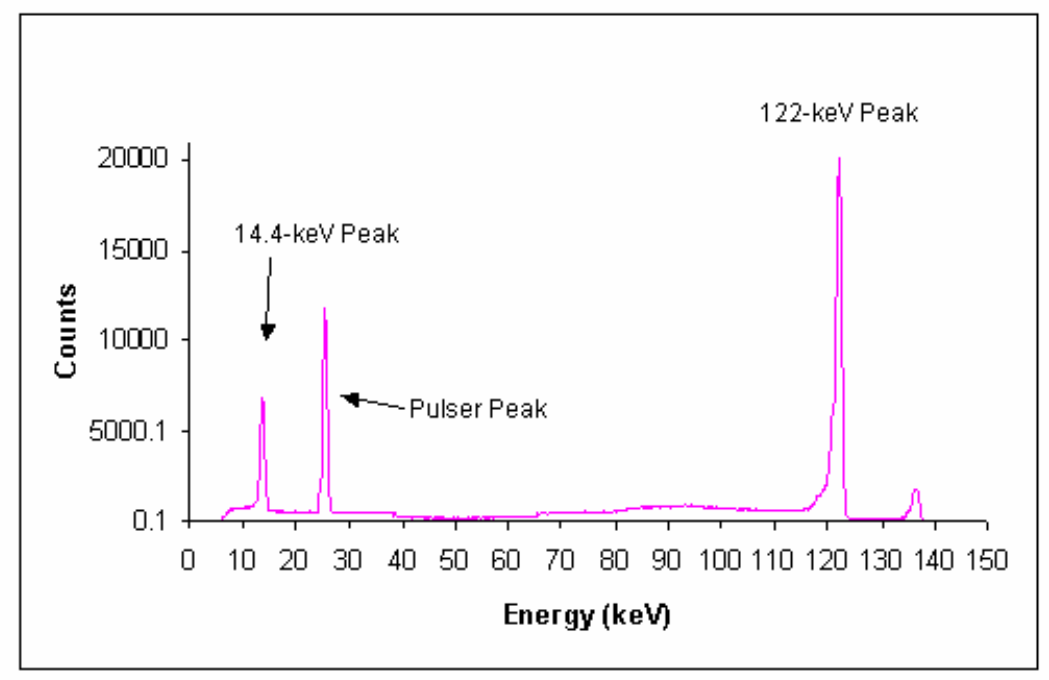

Figure 1 The spectrum demonstrates the viability of the detector fabrication technique. The detector has very low capacitance while maintaining a large area.

With these encouraging measurements made, and the detector fabrication technology clearly demonstrated, we planned to use this technology to make prototype XAS systems for our collaborators to test and demonstrate during Phase II. By the end of the Phase II, the PHDs Co. XAS detector systems would have been commercially available. Unfortunately, the Phase II was not funded. Consequently, this area of detector development has come to the end of the line for the present time. 